\title{
Tiling by incongruent equilateral triangles without requiring local finiteness
}

\begin{abstract}
Christian Richter*
Christian Richter absolvierte seine mathematische Ausbildung bis zur Habilitation an der Friedrich-Schiller-Universität Jena. Langjährige Forschungsaufenthalte verbrachte er am Institut de Mathématiques de Jussieu, Paris. Nach Ende seiner Förderung durch das Heisenberg-Programm der Deutschen Forschungsgemeinschaft arbeitet er
\end{abstract} wieder als Oberassistent an der Universität Jena.

Dedicated to Prof. Dr. Irmtraud Stephani on the occasion of her 75th birthday.

It has been shown by Tutte [7] that an equilateral triangle cannot be dissected into $n$ equilateral triangles of pairwise different sizes, $n \in\{2,3, \ldots\}$. A dissection into equilateral triangles of that kind is even impossible for arbitrary convex polygons (see [2, 8]).

Is there a tiling of the Euclidean plane by equilateral triangles all of different sizes? The answer to this question posed in [4, Exercise 2.4.10] and [3, Section C11] depends on what one means by a tiling: Scherer [6] proves that the plane cannot be dissected into incongruent equilateral triangles such that one of them is of minimal size. Tuza [8] notes that countably many equilateral triangles of different sizes can be packed into a given equilateral triangle such that only a remainder of measure zero is left uncovered. In the same way one can tile the whole plane except for a set of measure zero. Klaaßen [5]

\footnotetext{
* This research was supported by DFG grant RI 1087/3.
}

Kann man die euklidische Ebene mit paarweise inkongruenten gleichseitigen Dreiecken pflastern? Karl Scherer zeigte, dass eine solche Parkettierung kein kleinstes Dreieck enthalten kann. Bernhard Klaaßen fand eine Pflasterung der Ebene bis auf einen unbedeckten singulären Punkt, bei dem sich Dreiecke häufen. In der vorliegenden Arbeit wird die Ebene komplett parkettiert. Das vorgestellte Verfahren erlaubt sogar die Parkettierung einer jeden offenen Teilmenge der Ebene. Die benutzten Dreiecksanordnungen zeigen Häufungsphänomene. Der interessierte Leser ist deshalb eingeladen, über folgendes offene Problem nachzudenken: Besitzt die Ebene eine Pflasterung durch inkongruente gleichseitige Dreiecke, die sich in keinem Punkt der Ebene häufen? 


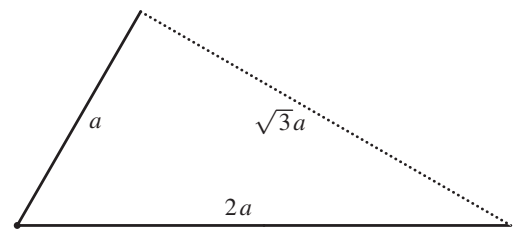

(a)

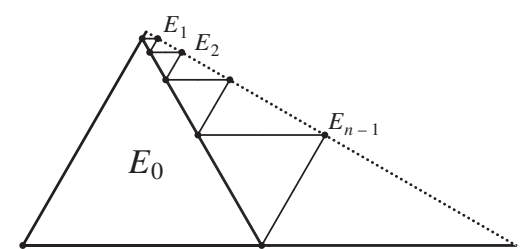

(b)

Fig. 1 (a) An auxiliary piece of size $a$, and (b) the process of its $n$-reduction

constructs a decomposition of the plane except for one singular point into incongruent equilateral triangles. Every neighbourhood of that point contains infinitely many pieces of the dissection, but the point itself is not covered. Moreover, Klaaßen gives a decomposition of an equilateral triangle except for three singular points.

The gap between dissections including a triangle of minimal size on the one hand and tilings allowing uncovered singular points on the other hand is large. The present note is devoted to a concept of tiling that admits accumulation phenomena, but does not accept uncovered points. The question for a locally finite tiling of the plane by incongruent equilateral triangles remains open.

Let $\mathbb{R}, \mathbb{Q}, \mathbb{Z}$, and $\mathbb{N}$ denote the sets of real, rational, integer and non-negative integer numbers, respectively. $\operatorname{cl}(S)$ stands for the closure of a set $S \subseteq \mathbb{R}^{2}$. A family $\mathcal{T}=\left\{T_{i}: i \in I\right\}$ of subsets of the plane $\mathbb{R}^{2}$ will be called a tiling of a set $S \subseteq \mathbb{R}^{2}$ if $S=\bigcup_{i \in I} T_{i}$ and the interiors of any two distinct members of $\mathcal{T}$ are disjoint (see e.g. [3, Chapter C]). A point $x \in \mathbb{R}^{2}$ is called singular with respect to $\mathcal{T}$ if every neighbourhood of $x$ meets infinitely many sets from $\mathcal{T}$ (see [4, Section 3.1]). We denote the set of all singular points of $\mathcal{T}$ by $\operatorname{sing}(\mathcal{T})$. A tiling $\mathcal{T}$ is called perfect if, for any two different tiles $T_{i_{1}}, T_{i_{2}}$, there exists a similarity transformation of the plane mapping $T_{i_{1}}$ onto $T_{i_{2}}$, but $T_{i_{1}}$ and $T_{i_{2}}$ are incongruent in the isometric sense (see [1]).

We are going to present a construction of perfect tilings for certain sets $S \subseteq \mathbb{R}^{2}$ by equilateral triangles. Within this paper triangles are understood to be closed sets. The size of an equilateral triangle is its edge length. Our construction is based on an auxiliary decomposition of $S$ into equilateral triangles and so-called auxiliary pieces. An auxiliary piece $A \subseteq \mathbb{R}^{2}$ is obtained by taking half of an equilateral triangle and removing its larger leg, including the end-points, as shown in Fig. 1(a). The size of $A$ is the length of the smaller leg. The missing leg $A^{b}=\operatorname{cl}(A) \backslash A$ is called the singular edge of $A$. In the illustrations all vertices belonging to the respective sets are emphasized and missing edges are dotted.

Theorem. Suppose a set $S \subseteq \mathbb{R}^{2}$ satisfies

$$
S=\bigcup_{A \in \mathcal{A}} A \cup \bigcup_{E \in \mathcal{E}} E=\bigcup_{A \in \mathcal{A}} \operatorname{cl}(A) \cup \bigcup_{E \in \mathcal{E}} E
$$

where $\mathcal{E}$ is a family of mutually incongruent equilateral triangles whose edge lengths belong to a set $\left\{c_{i} 2^{k}: i \in\{1, \ldots, m\}, k \in \mathbb{Z}\right\}$ with $m \in \mathbb{N}$ and positive real numbers $c_{i}$ and $\mathcal{A}$ is a family of auxiliary pieces such that the members of $\mathcal{A} \cup \mathcal{E}$ have pairwise disjoint interiors. 
Then $S$ admits a perfect tiling $\mathcal{T}$ by equilateral triangles satisfying

$$
\operatorname{sing}(\mathcal{T})=\operatorname{sing}(\mathcal{A} \cup \mathcal{E}) \cup \bigcup_{A \in \mathcal{A}} A^{b}
$$

Proof. It is enough to find families $\mathcal{E}_{A}, A \in \mathcal{A}$, of equilateral triangles with pairwise disjoint interiors such that for all $A \in \mathcal{A}$ one has

$$
A \subseteq \bigcup_{E \in \mathcal{E}_{A}} E \subseteq \operatorname{cl}(A), \quad \operatorname{sing}\left(\mathcal{E}_{A}\right)=A^{\mathrm{b}}
$$

and all triangles from $\mathcal{T}=\mathcal{E} \cup \bigcup_{A \in \mathcal{A}} \mathcal{E}_{A}$ are of different size. Then $\mathcal{T}$ is the required perfect tiling of $S$. Indeed, the tiling property is obvious. Moreover, if $x \in \mathbb{R}^{2}$ is singular with respect to $\mathcal{T}$, but not with respect to $\mathcal{A} \cup \mathcal{E}$, then there are finite subfamilies $\mathcal{E}^{\prime} \subseteq$ $\mathcal{E}$ and $\mathcal{A}^{\prime} \subseteq \mathcal{A}$ such that every sufficiently small open neighbourhood $U$ of $x$ satisfies $\{M \in \mathcal{A} \cup \mathcal{E}: U \cap M \neq \emptyset\} \subseteq \mathcal{A}^{\prime} \cup \mathcal{E}^{\prime}$ and therefore meets infinitely many triangles from $\bigcup_{A \in \mathcal{A}^{\prime}} \mathcal{E}_{A}$. Hence $x \in \operatorname{sing}\left(\mathcal{E}_{A_{0}}\right)=A_{0}^{b}$ for some $A_{0} \in \mathcal{A}^{\prime}$, which proves the inclusion $\subseteq$ in the claim concerning $\operatorname{sing}(\mathcal{T})$. Conversely, if $x \in \operatorname{sing}(\mathcal{A} \cup \mathcal{E}) \cup \bigcup_{A \in \mathcal{A}} A^{\text {b }}=\operatorname{sing}(\mathcal{A} \cup$ $\mathcal{E}) \cup \bigcup_{A \in \mathcal{A}} \operatorname{sing}\left(\mathcal{E}_{A}\right)$ then every neighbourhood $U$ of $x$ meets infinitely many members of $\mathcal{A} \cup \mathcal{E}$ and thus in particular infinitely many triangles from $\bigcup_{A \in \mathcal{A}} \mathcal{E}_{A} \cup \mathcal{E}=\mathcal{T}$ or there exists some $A_{0} \in \mathcal{A}$ such that $U$ meets infinitely many triangles from $\mathcal{E}_{A_{0}} \subseteq \mathcal{T}$. This yields $x \in \operatorname{sing}(T)$.

For defining the families $\mathcal{E}_{A}$ we introduce the process of $n$-reduction of an auxiliary piece $A$ of size $a$ for $n \in\{3,4, \ldots\}$ (see Fig. 1(b)): $A$ is dissected into $n$ equilateral triangles $E_{j}$, $0 \leq j \leq n-1$, whose edges are of length $\frac{2^{n}-2}{2^{n}-1} a$ if $j=0$ and $\frac{2^{j}}{2^{n}-1} a$ if $1 \leq j \leq n-1$, and $n$ auxiliary pieces $A_{j}^{\prime}$ of the sizes $\frac{2^{j}}{2^{n}-1} a, 0 \leq j \leq n-1$. The triangles $E_{j}$ are subsets of $\operatorname{cl}(A)$ and have different sizes. The singular edge of $A$ is the union of the singular edges of the auxiliary pieces $A_{j}^{\prime}, 0 \leq j \leq n-1$. We call the sets $A_{j}^{\prime}$ successors of degree 1 of $A$. Since $n \geq 3$, their sizes are bounded by $\frac{2^{3-1}}{2^{3}-1} a=\frac{4}{7} a$.

We iterate this procedure. Given all successors of degree $d-1$ of $A$, say $A_{1}^{\prime}, \ldots, A_{l}^{\prime}$, we choose suitable numbers $n_{1}^{\prime}, \ldots, n_{l}^{\prime} \in\{3,4, \ldots\}$ and perform the processes of $n_{j}^{\prime}$ reduction of $A_{j}^{\prime}, 1 \leq j \leq l$. This way we obtain $n_{1}^{\prime}+\ldots+n_{l}^{\prime}$ new equilateral triangles and the same number of successors of degree 1 of $A_{1}^{\prime}, \ldots, A_{l}^{\prime}$, that we call successors of degree $d$ of $A$.

The family $\mathcal{E}_{A}$ of all emerging equilateral triangles in the processes of reduction of $A$ as well as of all its successors of arbitrary degree $d \geq 1$ consists of triangles with disjoint interiors. The reductions of $A$ and of all its successors of degree smaller than $d$ produce finitely many triangles in $\mathrm{cl}(A)$ that cover $A$ except for the interiors of its successors of degree $d$. The sizes of that successors are not larger than $\left(\frac{4}{7}\right)^{d} a$ and the union of their singular edges is the singular edge $A^{b}$ of $A$. Therefore $A \subseteq \bigcup_{E \in \mathcal{E}_{A}} E \subseteq \operatorname{cl}(A)$ and $\operatorname{sing}\left(\mathcal{E}_{A}\right)=A^{b}$. It remains to prove that in every processes of reduction of an auxiliary piece $A$ from $\mathcal{A}$ itself or from the family of all successors of arbitrary degree of sets from $\mathcal{A}$ one can choose an appropriate number $n=n(A)$ of occurring triangles such that all members of the final tiling $\mathcal{T}=\mathcal{E} \cup \bigcup_{A \in \mathcal{A}} \mathcal{E}_{A}$ of $S$ are of different size. 
The family $\mathcal{A}$ is at most countable. (For every $A \in \mathcal{A}$ we can fix a point $x_{A}$ with rational coordinates in the interior of $A$. This defines an injective map from $\mathcal{A}$ into $\mathbb{Q}^{2}$.) Let $\mathcal{A}=$ $\left\{A_{1}, A_{2}, \ldots\right\}$. (A similar treatment is possible if $\mathcal{A}$ is finite.)

The reductions will be performed in the following order: First we reduce $A_{1}$, next $A_{2}$ and the successors of degree 1 of $A_{1}$, then $A_{3}$, the successors of degree 1 of $A_{2}$ and the successors of degree 2 of $A_{1}$ and so on. This way all reductions are done consecutively. Suppose that $r \geq 0$ reduction steps are already performed. Let $A$ be the next auxiliary piece that is to reduce. This has to be done in such a way that the sizes of the resulting equilateral triangles differ from those of the triangles from $\mathcal{E}$ and of the finitely many, say $s$, triangles that have been obtained by the $r$ previous reduction steps. According to the assumption concerning $\mathcal{E}$ all forbidden sizes belong to some set $C=\left\{c_{i} 2^{k}: i \in\left\{1, \ldots, m^{\prime}\right\}, k \in \mathbb{Z}\right\}$, where $m^{\prime} \in \mathbb{N}, m^{\prime} \leq m+s$, and $c_{i}>0$. We put $C_{i}=\left\{c_{i} 2^{k}: k \in \mathbb{Z}\right\}, 1 \leq i \leq m^{\prime}$.

Next we show that, for every $i \in\left\{1, \ldots, m^{\prime}\right\}$, there exists at most one number $n=n(i) \in$ $\{3,4, \ldots\}$ such that $n$-reduction of $A$ produces a resulting triangle whose size is in $C_{i}$ : Suppose that $T_{1}$ and $T_{2}$ are triangles with sizes in $C_{i}$ that result from $n_{1}$-reduction and $n_{2}$-reduction of $A$, respectively. Their respective sizes must satisfy $s_{1}=c_{i} 2^{k_{1}}, s_{2}=c_{i} 2^{k_{2}}$ for suitable $k_{1}, k_{2} \in \mathbb{Z}$, say $k_{1} \leq k_{2}$, and

$$
\begin{aligned}
& s_{1} \in\left\{\frac{2^{n_{1}}-2}{2^{n_{1}}-1} a\right\} \cup\left\{\frac{2^{j_{1}}}{2^{n_{1}}-1} a: 1 \leq j_{1} \leq n_{1}-1\right\}, \\
& s_{2} \in\left\{\frac{2^{n_{2}}-2}{2^{n_{2}}-1} a\right\} \cup\left\{\frac{2^{j_{2}}}{2^{n_{2}}-1} a: 1 \leq j_{2} \leq n_{2}-1\right\},
\end{aligned}
$$

where $a$ is the size of $A$. Consequently, $s_{2}=2^{k_{2}-k_{1}} s_{1}$ and

$$
\begin{array}{llll}
\frac{2^{n_{2}}-2}{2^{n_{2}}-1}=\frac{\left(2^{n_{1}}-2\right) 2^{k_{2}-k_{1}}}{2^{n_{1}}-1} & \text { if } & s_{1}=\frac{2^{n_{1}}-2}{2^{n_{1}}-1} a, & s_{2}=\frac{2^{n_{2}}-2}{2^{n_{2}}-1} a, \\
\frac{2^{n_{2}}-2}{2^{n_{2}}-1}=\frac{2^{j_{1}+k_{2}}-k_{1}}{2^{n_{1}}-1} & \text { if } & s_{1}=\frac{2^{j_{1}}}{2^{n_{1}}-1} a, & s_{2}=\frac{2^{n_{2}}-2}{2^{n_{2}}-1} a, \\
\frac{2^{j_{2}}}{2^{n_{2}}-1}=\frac{\left(2^{n_{1}}-2\right) 2^{k_{2}-k_{1}}}{2^{n_{1}}-1} & \text { if } & s_{1}=\frac{2^{n_{1}}-2}{2^{n_{1}}-1} a, & s_{2}=\frac{2^{j_{2}}}{2^{n_{2}}-1} a, \\
\frac{2^{j_{2}}}{2^{n_{2}}-1}=\frac{2^{j_{1}+k_{2}-k_{1}}}{2^{n_{1}}-1} & \text { if } & s_{1}=\frac{2^{j_{1}}}{2^{n_{1}}-1} a, & s_{2}=\frac{2^{j_{2}}}{2^{n_{2}}-1} a .
\end{array}
$$

In any of the four cases we obtain an equality of two completely canceled fractions. Hence their denominators agree. This gives $2^{n_{2}}-1=2^{n_{1}}-1$, which shows that $n_{1}=n_{2}=n(i)$ is unique.

The last observation tells us that there exist at most $m^{\prime}$ numbers $n \in\{3,4, \ldots\}$ such that $n$-reduction of $A$ produces a triangle of forbidden size. Thus we can pick some $n(A) \in\{3,4, \ldots\}$ such that the $n(A)$-reduction of $A$ results in equilateral triangles of sizes different from the previously given triangles.

This shows that there exists a sequence of consecutive reductions that lead to a perfect tiling $\mathcal{T}$. The proof is complete. 


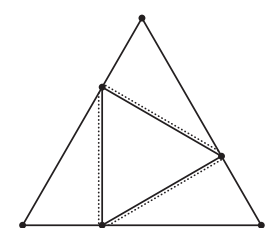

(a)

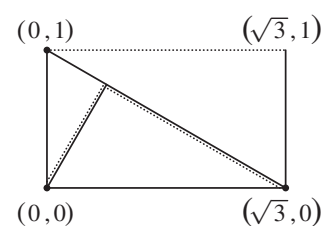

(b)

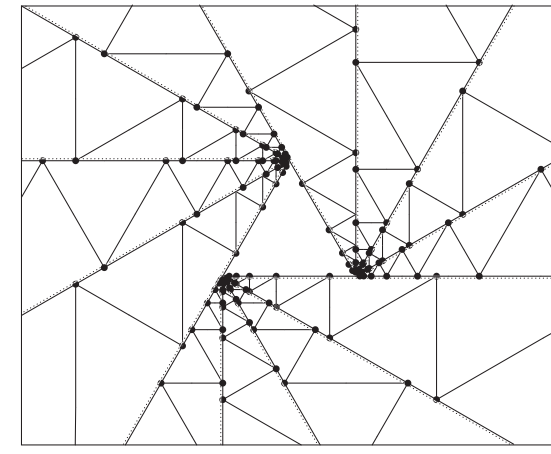

(c)

Fig. 2 Auxiliary decompositions (a) of an equilateral triangle, (b) of the rectangle $R^{H}$, and (c) of the whole plane

The assumption $S=\bigcup_{A \in \mathcal{A}} \operatorname{cl}(A) \cup \bigcup_{E \in \mathcal{E}} E$ in the theorem is a necessary consequence of $S=\bigcup_{A \in \mathcal{A}} A \cup \bigcup_{E \in \mathcal{E}} E$ if $S$ is closed. In that situation we obtain the following.

Corollary 1. Suppose a closed set $F \subseteq \mathbb{R}^{2}$ is of the form $F=\bigcup_{A \in \mathcal{A}} A \cup \bigcup_{E \in \mathcal{E}} E$ where $\mathcal{E}$ is a family of mutually incongruent equilateral triangles whose edge lengths belong to a set $\left\{c_{i} 2^{k}: i \in\{1, \ldots, m\}, k \in \mathbb{Z}\right\}$ with $m \in \mathbb{N}$ and positive real numbers $c_{i}$ and $\mathcal{A}$ is $a$ family of auxiliary pieces such that the members of $\mathcal{A} \cup \mathcal{E}$ have pairwise disjoint interiors. Then $F$ admits a perfect tiling by equilateral triangles.

Corollary 1 provides a perfect tiling of an equilateral triangle $E$ by countably many equilateral triangles: Fig. 2 (a) illustrates an auxiliary decomposition $\mathcal{A} \cup \mathcal{E}$ of $E$ consisting of three auxiliary pieces and one equilateral triangle.

Note that every triangle $T$ admitting a tiling by equilateral triangles is necessarily equilateral: Every vertex of $T$ must belong to some tile. Hence every inner angle of $T$ has a size of at least $\frac{\pi}{3}$.

When does a (closed) polygon $P \subseteq \mathbb{R}^{2}$ admit a perfect tiling by equilateral triangles? Does it suffice that the sizes of all inner angles of $P$ are at least $\frac{\pi}{3}$ ?

Corollary 2. Every open set $G \subseteq \mathbb{R}^{2}$ admits a perfect tiling by equilateral triangles.

Proof. Let $R_{0}$ be the closed rectangle with vertices $(0,0),(\sqrt{3}, 0),(\sqrt{3}, 1)$, and $(0,1)$. The half-open rectangle $R^{H}$ is defined as $R^{H}=R_{0} \backslash\{(\lambda, 1): 0<\lambda \leq \sqrt{3}\}$ (see Fig. 2(b)). The families $\mathcal{F}_{n}=\left\{2^{-n}\left(R^{H}+(k \sqrt{3}, l)\right): k, l \in \mathbb{Z}\right\}, n \in \mathbb{N}$, of shifted and dilated copies of $R^{H}$ are tilings of the plane $\mathbb{R}^{2}$. If two rectangles from $\bigcup_{n=0}^{\infty} \mathcal{F}_{n}$ have common interior points then one of the interiors is completely contained in the other one. We define $\mathcal{G}_{0}=\left\{R \in \mathcal{F}_{0}: \operatorname{cl}(R) \subseteq G\right\}$ and inductively $\mathcal{G}_{n+1}=\mathcal{G}_{n} \cup\left\{R \in \mathcal{F}_{n+1}: \operatorname{cl}(R) \subseteq\right.$ $G$ and the interior of $R$ is disjoint with all members from $\left.\mathcal{G}_{n}\right\}$. The family $\mathcal{G}=\bigcup_{n=0}^{\infty} \bar{G}_{n}$ is a tiling of $G$, since $G$ is open, and every half-open rectangle $R \in \mathcal{G}$ satisfies $\operatorname{cl}(R) \subseteq G$. 
Every $R \in \mathcal{G}$ can be dissected into three auxiliary pieces $A_{1}(R), A_{2}(R), A_{3}(R)$, as is illustrated in Fig. 2(b). Then $\mathcal{A}=\left\{A_{i}(R): R \in \mathcal{G}, i \in\{1,2,3\}\right\}$ is a tiling of $G$ by auxiliary pieces such that, moreover, $\operatorname{cl}(A)=\operatorname{cl}\left(A_{i}(R)\right) \subseteq \operatorname{cl}(R) \subseteq G$ for every $A=$ $A_{i}(R) \in \mathcal{A}$. Hence the theorem can be applied to $S=G, \mathcal{E}=\emptyset$ and that very $\mathcal{A}$. This yields the claim.

Corollary 2 provides a perfect tiling $\mathcal{T}$ of the plane $G=\mathbb{R}^{2}$ by equilateral triangles. In that particular situation the proof of the corollary gives $\mathcal{G}=\mathcal{G}_{0}=\mathcal{F}_{0}$ and the decomposition $\mathcal{A}$ is obtained by dissecting all half-open rectangles from $\mathcal{F}_{0}$ into three auxiliary pieces as in Fig. 2(b). The dotted lines in the figure represent the singularities of $\mathcal{T}$ in the respective rectangle, as is stated by the theorem. So the set of all singularities is the union of countably many line segments and cannot be covered by finitely many straight lines.

Fig. 2(c) illustrates an application of the theorem (or of Corollary 1) resulting in a perfect tiling of the plane $\mathbb{R}^{2}$ whose set of singularities is the union of 12 half-lines: We fix real numbers $c_{0}, \ldots, c_{12}>0$ such that $\frac{c_{i_{1}}}{c_{i_{2}}} \notin\left\{2^{k}: k \in \mathbb{Z}\right\}$ for $0 \leq i_{1}<i_{2} \leq 12$. We split the plane into an equilateral triangle $E_{0}$ of size $c_{0}$ and 12 half-open cones $C_{1}, \ldots, C_{12}$ with opening angle $\frac{\pi}{6}$, whose apices are the vertices of $E_{0} . E_{0}$ and $C_{1}, \ldots, C_{12}$ are pairwise disjoint. Next we dissect each cone $C_{i}$ into a sequence of equilateral triangles $E_{i}^{(k)}$ and auxiliary pieces $A_{i}^{(k)}$ of sizes $c_{i} 2^{k}, k \in \mathbb{Z}$. (Strictly speaking, one vertex of every $E_{i}^{(k)}$ is on the missing side of $C_{i}$.) The singular edges of the auxiliary pieces $A_{i}^{(k)}$ are subsets of the missing side of $C_{i}$. Then the families $\mathcal{A}=\left\{A_{i}^{(k)}: 1 \leq i \leq 12, k \in \mathbb{Z}\right\}$ and $\mathcal{E}=\left\{E_{0}\right\} \cup\left\{E_{i}^{(k)}: 1 \leq i \leq 12, k \in \mathbb{Z}\right\}$ satisfy the assumptions of the theorem, which in turn gives a perfect tiling of the plane by equilateral triangles whose set of singularities is the union of the 12 missing sides of the cones $C_{i}$.

How small can the set of singularities of a perfect tiling of the Euclidean plane by equilateral triangles be? Can it be countable, discrete, finite or even empty?

We have found a perfect tiling of an equilateral triangle by equilateral triangles whose set of singularities is the union of three line segments (see Fig. 2(a)). Can an equilateral triangle be perfectly tiled by countably many equilateral triangles such that there are only countably many or even finitely many singularities?

\section{References}

[1] Brooks, R.L.; Smith, C.A.B.; Stone, A.H.; Tutte, W.T.: The dissection of rectangles into squares. Duke Math. J. 7 (1940), 312-340.

[2] Buchman, E.: The impossibility of tiling a convex region with unequal equilateral triangles. Amer. Math. Monthly 88 (1981), 748-753.

[3] Croft, H.T.; Falconer, K.J.; Guy, R.K.: Unsolved problems in geometry. Problem Books in Mathematics. Unsolved Problems in Intuitive Mathematics, II. Springer-Verlag, New York 1991.

[4] Grünbaum, B.; Shephard, G.C.: Tilings and patterns. W.H. Freeman and Company, New York 1987.

[5] Klaaßen, B.: Infinite perfekte Dreieckszerlegungen auch für gleichseitige Dreiecke. Elem. Math. 50 (1995), 116-121.

[6] Scherer, K.: The impossibility of a tesselation of the plane into equilateral triangles whose sidelengths are mutually different, one of them being minimal. Elem. Math. 38 (1983), 1-4. 
[7] Tutte, W.T.: The dissection of equilateral triangles into equilateral triangles. Proc. Cambridge Philos. Soc. 44 (1948), 463-482.

[8] Tuza, Z.: Dissections into equilateral triangles. Elem. Math. 46 (1991), 153-158.

\section{Christian Richter}

Mathematical Institute

Friedrich Schiller University

D-07737 Jena, Germany

e-mail: christian.richter@uni-jena.de 